\title{
A Double Sigmoid-Based Beamforming Algorithm With Reduced Computational Complexity
}

\author{
Raimundo N. G. Robert, Ciro André Pitz, and Rui Seara
}

\begin{abstract}
This paper introduces a new adaptive beamforming algorithm for cellular systems. Such an algorithm is based on the adaptive combination of vector projections (ACVP) framework presented in the open literature. The main novelty of the proposed algorithm is the use of both unipolar and bipolar sigmoid functions for supporting the adjustment of the combination coefficients used in the ACVP framework. The degree of freedom of these sigmoid functions allows updating the beamforming coefficients by using a smaller number of vector projections. As a consequence, the proposed algorithm, termed here double sigmoid ACVP (DS-ACVP), presents a low computational complexity and is capable of outperforming other adaptive beamforming algorithms from the literature. Numerical simulation results corroborate the effectiveness of the proposed approach.
\end{abstract}

Index Terms-Adaptive algorithm, adaptive antenna arrays, beamforming, mobile communications, stochastic gradient method.

\section{INTRODUCTION}

A NTENNA arrays have been proven to be a powerful approach for increasing the capacity of cellular systems [1][3]. The spatial filtering capability of the antenna arrays can be exploited by beamforming algorithms aiming to reduce the interference levels and strengthen the signal of interest (SOI) in both uplink and downlink channels. Moreover, considering adaptive beamforming algorithms, a real-time adjustment of the radiation pattern can be achieved, leading to higher levels of signal-to-interference-plus-noise ratio (SINR) [4].

Most adaptive beamforming algorithms consider the availability of a training sequence for its proper operation. In the conventional approach discussed in [5], the error signal resulting from the difference between the training signal and the array output is used to control the beamforming algorithm. The major drawback of this strategy is its dependence on high levels of correlation between the SOI and the training signal, which is a hard condition to be fulfilled in cellular systems [6]. In this context, most recent beamforming approaches are based on a priori knowledge of the channel state information (CSI), which is estimated by using training sequences. For instance, efficient schemes for distributed transmit beamforming are derived in [7] by means of an optimal training sequence design. In [8], a reduced-dimension training sequence is proposed for estimating the downlink CSI and obtaining a hybrid

Raimundo N. G. Robert, Ciro André Pitz, and Rui Seara are with the LINSE-Laboratory of Circuits and Signal Processing, Department of Electrical and Electronic Engineering, Federal University of Santa Catarina, Florianópolis, SC, Brazil. E-mails: \{nonato, ciropitz, seara\}@linse.ufsc.br.

This work was supported in part by the National Council for Scientific and Technological Development (CNPq).

Digital Object Identifier: 10.14209/jcis.2019.13 analog-digital beamforming in large-scale antenna systems. In addition, CSI feedback mechanisms are discussed in [9] and [10] in order to compute the downlink transmit beamforming in frequency division duplex (FDD) systems. Another common strategy used in adaptive beamforming is to minimize the antenna array output power subject to array gain constraints in the SOI direction [11]. Specifically, the beamforming algorithms of this class are derived from the minimum variance criterion [12], [13], thus requiring an accurate estimate of both the input autocorrelation matrix and the direction-of-arrival (DOA) of the SOI [14], [15]. As a consequence, even small errors in the estimation process may significantly reduce the performance of the algorithms based on this strategy [15]. Aiming to overcome this problem, algorithms such as those discussed in [16]-[21] were developed to provide robustness against estimation errors.

Some algorithms discussed in the open literature aim to reduce the additional resources required for either providing a training signal or estimating the CSI, the input autocorrelation matrix, and the DOA. Such algorithms provide both high SINR performance and low computational burden by exploiting some particular aspects of mobile communication systems. For instance, the algorithms introduced in [22]-[24] only use the signals already available at the input and output of the correlators in code-division-multiple-access (CDMA) systems. On the other hand, the constrained stochastic gradient (CSG) algorithms [25] and its improved version (ICSG) [26] consider the space-time equalization structure discussed in [27], aiming to obtain the downlink beamforming from the uplink data symbols. The implementation challenges of the CSG-type beamforming algorithms are overcome in [28] by introducing the adaptive-projection CSG (AP-CSG), which yields lower computational complexity and higher SINR levels than both CSG and ICSG algorithms. Furthermore, the adaptiveprojection approach presented in [28] is used in [29] to derive a new form of quadratically-constrained minimization problem. As a result, the adaptive-projection quadratically-constrained stochastic gradient (AP-QCSG) algorithm is developed, improving the SINR performance in realistic scenarios formed by spatially distributed sources [29].

In [30], a new framework for adaptive beamforming design is derived from a unifying view on the mean behavior of adaptive-projection CSG-type algorithms. Such a framework, termed adaptive combination of vector projections (ACVP), is introduced in [30] to devise the sigmoid-based ACVP (SB-ACVP) beamforming algorithm, which applies a unipolar sigmoid function for dynamically adjusting the linear combination in the ACVP framework. Such as the CSG-type beamforming algorithms, the SB-ACVP considers a space- 
time equalization structure [27] to separate the SOI from the interferences. The disadvantage of such an approach is that it requires a minimum initial SINR for detecting the data symbols related to the SOI from the uplink signal. However, such a minimum SINR can be reduced by adaptive modulation and coding schemes [31], [32], which allow the use of loworder modulation and low-rate coding at the beginning of the communication session. As shown in [30], the SB-ACVP outperforms competing algorithms from the open literature and presents a better trade-off between performance and computational cost. Thus, taking into account the satisfactory performance of the SB-ACVP, the present paper proposes a novel adaptive beamforming algorithm also based on the ACVP framework. In contrast to the SB-ACVP, the proposed algorithm, termed here double sigmoid ACVP (DS-ACVP), obtains the linear combination coefficients using both unipolar and bipolar sigmoid functions, allowing to reduce the number of vector projections required in the beamforming update. As a consequence, the proposed DS-ACVP presents high SINR performance with lower computational complexity as compared with the SB-ACVP.

This paper is organized as follows. Section II presents the theoretical basis, including both system model and problem statement. Section III revisits the ACVP framework and the SB-ACVP algorithm. Section IV presents the proposed DS-ACVP algorithm. Section V shows numerical simulation results aiming to assess the performance of the proposed DS-ACVP algorithm. Finally, Section VI presents concluding remarks.

\section{System Model and Problem Statement}

The scenario considered here involves a linear array with $K$ antennas located at the base station (BS) and $M$ users that share the same channel. In this scenario, the baseband signal received at the $\mathrm{BS}$ is arranged in the input vector $\mathbf{x}(n) \in$ $\mathbb{C}^{K \times 1}$, which is given by

$$
\mathbf{x}(n)=\sum_{m=1}^{M} \mathbf{s}_{m}(n)+\mathbf{r}(n)
$$

with $\mathbf{s}_{m}(n)$ and $\mathbf{r}(n) \in \mathbb{C}^{K \times 1}$ representing, respectively, the signal corresponding to the $m$ th user and the additive white Gaussian noise (AWGN) present in the antennas. By assuming $L$ independent paths between each user and the $\mathrm{BS}, \mathbf{s}_{m}(n)$ can be modeled as

$$
\mathbf{s}_{m}(n)=\mathbf{H}_{m} \mathbf{v}_{m}(n)
$$

where $\mathbf{v}_{m}(n) \in \mathbb{C}^{L \times 1}$ denotes the vector containing the symbols coming from each multipath, and $\mathbf{H}_{m} \in \mathbb{C}^{K \times L}$ represents the spatial response matrix whose column $\mathbf{h}_{m, l}$ denotes the steering vector of the signal arriving from the $l$ th multipath between the $m$ th mobile terminal and the BS [2].

The antenna array output, represented by $y(n)$, is expressed as the inner product between the beamforming vector $\mathbf{w}(n) \in$ $\mathbb{C}^{K \times 1}$ and the input vector $\mathbf{x}(n)$, i.e.,

$$
y(n)=\mathbf{w}^{\mathrm{H}}(n) \mathbf{x}(n) .
$$

Then, aiming to evaluate the SINR provided by $\mathbf{w}(n)$, the variance of $y(n)$ is obtained as

$$
\sigma_{y}^{2}(n)=\mathrm{E}\left[|y(n)|^{2}\right]=\mathbf{w}^{\mathrm{H}}(n) \mathbf{R}_{\mathbf{x}} \mathbf{w}(n)
$$

where $\mathbf{R}_{\mathbf{x}}=\mathrm{E}\left[\mathbf{x}(n) \mathbf{x}^{\mathrm{H}}(n)\right]$ denotes the input autocorrelation matrix.

Now, assuming that the symbols coming from each multipath are independent, i.e.,

$$
\mathrm{E}\left[\mathbf{v}_{m}(n) \mathbf{v}_{m}^{\mathrm{H}}(n)\right]=\frac{P_{m}}{L} \mathbf{I}_{L}
$$

with $\mathbf{I}_{L}$ and $P_{m}$ characterizing, respectively, the identity matrix with dimension $L \times L$ and the power of the received symbols, (4) can be rewritten as

$$
\sigma_{y}^{2}(n)=\sum_{m=1}^{M} P_{m} \mathbf{w}^{\mathrm{H}}(n) \mathbf{R}_{m} \mathbf{w}(n)+\sigma_{r}^{2}\|\mathbf{w}(n)\|^{2}
$$

where $\mathbf{R}_{m}(n)=\mathbf{H}_{m} \mathbf{H}_{m}^{\mathrm{H}} / L$ denotes the spatial covariance matrix of the $m$ th mobile terminal and $\sigma_{r}^{2}$ is the noise variance. Next, assuming that $m=1$ corresponds to the SOI, the interference-plus-noise power can be separated from the SOI power in (6), resulting in the following SINR expression:

$$
\Gamma(n)=\frac{\mathbf{w}^{\mathrm{H}}(n) \mathbf{R}_{\mathrm{soi}} \mathbf{w}(n)}{\mathbf{w}^{\mathrm{H}}(n) \mathbf{R}_{\mathrm{int}} \mathbf{w}(n)+\sigma_{r}^{2}\|\mathbf{w}(n)\|^{2}}
$$

with $\mathbf{R}_{\text {soi }}=P_{1} \mathbf{R}_{1}$ and $\mathbf{R}_{\text {int }}=\sum_{m=2}^{M} P_{m} \mathbf{R}_{m}$ denoting the correlation matrices corresponding to the SOI and to the interference, respectively.

The CSG-type algorithms presented in [25], [26], [28], and [29] operate based on the availability of both SOI and interference-plus-noise signal. As discussed in [27], a spacetime equalization structure can be used to estimate the SOI from $\mathbf{x}(n)$. Then, defining $\mathbf{d}(n) \in \mathbb{C}^{K \times 1}$, which contains the SOI snapshots at each antenna of the array, and subtracting it from $\mathbf{x}(n)$, a vector $\mathbf{z}(n)=\mathbf{x}(n)-\mathbf{d}(n)$ composed of snapshots of the interference-plus-noise signal is obtained. Thus, with the availability of both $\mathbf{d}(n)$ and $\mathbf{z}(n)$, the instantaneous SINR given by

$$
\hat{\Gamma}(n)=\frac{\mathbf{w}^{\mathrm{H}}(n) \mathbf{d}(n) \mathbf{d}^{\mathrm{H}}(n) \mathbf{w}(n)}{\mathbf{w}^{\mathrm{H}}(n) \mathbf{z}(n) \mathbf{z}^{\mathrm{H}}(n) \mathbf{w}(n)}
$$

is used as the objective function of the CSG-type beamforming algorithms. Specifically, the CSG [25] and the ICSG [26] are devised aiming to maximize (8) in two stages: in the first, the denominator of (8) is kept fixed while the numerator is maximized; in the second, the denominator is minimized keeping the numerator fixed. As a drawback, both CSG and ICSG require the individual estimation of each interfering signal, which is not an easy task due to the low power levels of the interferers. In contrast, the AP-CSG algorithm [28] is developed in order to eliminate the need for individual estimation of the interfering signals. To this end, the denominator of (8) is minimized while an adaptive-projection constraint is used for steering $\mathbf{w}(n)$ toward the SOI subspace [28]. Regarding the AP-QCSG [29], the approach consists of replacing the affine adaptive projection constraint considered in [28] by a more effective quadratic adaptive constraint, which allows exploiting all subspace spanned by the SOI. 


\section{REVIEW OF THE ACVP FRAMEWORK}

The ACVP framework proposed in [30] is based on a geometric interpretation of both AP-CSG and AP-QCSG algorithms. Such a geometric interpretation is obtained from the stochastic model describing the mean-weight behavior of these algorithms. In the case of the AP-CSG, the mean-weight behavior expression is written as

$$
\begin{aligned}
\mathrm{E}[\mathbf{w}(n+1)] & =\mathrm{E}[\mathbf{w}(n)]-\mu_{1} \mathbf{R}_{\text {int }} \mathrm{E}[\mathbf{w}(n)] \\
& +\mu_{2} \underline{\mathbf{R}}_{\mathrm{soi}} \mathrm{E}[\mathbf{w}(n)]+\mu_{1} \underline{\mathbf{R}}_{\mathrm{soi}} \mathbf{R}_{\text {int }} \mathrm{E}[\mathbf{w}(n)]
\end{aligned}
$$

where $\mu_{1}$ and $\mu_{2}$ denote the step-size parameters, and $\underline{\mathbf{R}}_{\text {soi }}$ is the normalized autocorrelation matrix of the SOI, given by [18]

$$
\underline{\mathbf{R}}_{\mathrm{soi}}=\mathrm{E}\left[\frac{\mathbf{d}(n) \mathbf{d}^{\mathrm{H}}(n)}{\|\mathbf{d}(n)\|^{2}}\right] .
$$

As shown in [26], such a matrix can be analytically obtained from the eigendecomposition of $\mathbf{R}_{\mathrm{soi}}$.

Regarding the AP-QCSG, the mean-weight behavior is modeled as follows [30]:

$$
\begin{aligned}
& \mathrm{E}[\mathbf{w}(n+1)]=\mathrm{E}[\mathbf{w}(n)]-\mu_{1} \mathbf{R}_{\text {int }} \mathrm{E}[\mathbf{w}(n)] \\
& \quad+\mathrm{E}[\chi(n)] \underline{\mathbf{R}}_{\mathrm{soi}} \mathrm{E}[\mathbf{w}(n)]+\frac{\mu_{1}}{2} \underline{\mathbf{R}}_{\mathrm{soi}} \mathbf{R}_{\text {int }} \mathrm{E}[\mathbf{w}(n)]
\end{aligned}
$$

with

$$
\begin{gathered}
\mathrm{E}[\chi(n)] \cong \frac{1}{2} \mu_{1} \frac{\mathrm{E}\left[G_{\mathbf{d z}}^{*}(n)\right]}{\mathrm{E}\left[G_{\mathbf{d}}(n)\right]}+\frac{\sqrt{\mathrm{E}[\Delta(n)]}}{\mathrm{E}\left[G_{\mathbf{d}}(n)\right]}-1 \\
\mathrm{E}[\Delta(n)] \cong\left(1+\mu_{2}\right)\left\{\mathrm{E}\left[G_{\mathbf{d}}(n)\right]\right\}^{2}-\mu_{1}^{2} \operatorname{Im}\left\{\mathrm{E}\left[G_{\mathbf{d z}}(n)\right]\right\} \\
\mathrm{E}\left[G_{\mathbf{d}}(n)\right]=\mathrm{E}\left[\mathbf{w}^{\mathrm{H}}(n)\right] \mathbf{R}_{\mathrm{soi}} \mathrm{E}[\mathbf{w}(n)]
\end{gathered}
$$

and

$$
\mathrm{E}\left[G_{\mathbf{d z}}(n)\right]=\mathrm{E}\left[\mathbf{w}^{\mathrm{H}}(n)\right] \mathbf{R}_{\text {soi }} \mathbf{R}_{\text {int }} \mathrm{E}[\mathbf{w}(n)] .
$$

It is shown in [30] that the mean-weight behavior of both AP-CSG and AP-QCSG algorithms are somewhat similar. More specifically, one verifies from (9) and (11) that such algorithms operate based on the linear combination of $\mathbf{v}_{1}(n)=\mathbf{R}_{\text {int }} \mathrm{E}[\mathbf{w}(n)], \mathbf{v}_{2}(n)=\underline{\mathbf{R}}_{\mathrm{soi}} \mathrm{E}[\mathbf{w}(n)]$, and $\mathbf{v}_{3}(n)=$ $\underline{\mathbf{R}}_{\mathrm{soi}} \mathbf{R}_{\text {int }} \mathrm{E}[\mathbf{w}(n)]$. In the case of the AP-CSG, the linear combination is fixed, with the coefficients given, respectively, by $-\mu_{1}, \mu_{2}$, and $\mu_{1}$. On the other hand, the AP-QCSG algorithm performs the linear combination by using fixed coefficients for $\mathbf{v}_{1}(n)$ and $\mathbf{v}_{3}(n)\left(-\mu_{1}\right.$ and $\mu_{2} / 2$, respectively), whereas the coefficient corresponding to $\mathbf{v}_{2}(n)$ is given by the timevarying term $\mathrm{E}[\chi(n)]$. Thus, taking into account the difference of performance between the considered algorithms [29], the idea behind the ACVP framework is to dynamically adjust the linear combination of $\mathbf{v}_{1}(n), \mathbf{v}_{2}(n)$, and $\mathbf{v}_{3}(n)$ aiming to enhance the SINR levels at the antenna array output [30].

The general update expression of the ACVP framework is derived in [30] balancing the individual contribution of the vectors on the right-hand side of (9) and (11). To this end, $\mathbf{v}_{1}(n)$ is replaced by $\overline{\mathbf{v}}_{1}(n)=\underline{\mathbf{R}}_{\text {int }} \mathrm{E}[\mathbf{w}(n)]$, with

$$
\underline{\mathbf{R}}_{\mathrm{int}}=\mathrm{E}\left[\frac{\mathbf{z}(n) \mathbf{z}^{\mathrm{H}}(n)}{\|\mathbf{z}(n)\|^{2}}\right]
$$

denoting the normalized version of $\mathbf{R}_{\mathrm{int}}$. Since the sum of the eigenvalues of $\underline{\mathbf{R}}_{\text {int }}$ is unitary (normalized matrix), one can verify that $\left\|\overline{\mathbf{v}}_{1}(n)\right\| \leq \| \mathrm{E}[\mathbf{w}(n) \|$. This upper bound is also obtained for $\mathbf{v}_{2}(n)$ as a result of the normalized matrix $\underline{\mathbf{R}}_{\text {soi }}$. Concerning $\mathbf{v}_{3}(n)$, its balanced version is given by $\overline{\mathbf{v}}_{3}(n)=$ $\underline{\mathbf{R}}_{\mathrm{soi}} \underline{\mathbf{R}}_{\text {int }} \mathrm{E}[\mathbf{w}(n)]$, resulting in $\left\|\overline{\mathbf{v}}_{3}(n)\right\| \leq \| \mathrm{E}[\mathbf{w}(n) \|$. Then, considering $\overline{\mathbf{v}}_{1}(n), \overline{\mathbf{v}}_{2}(n)=\mathbf{v}_{2}(n)$, and $\overline{\mathbf{v}}_{3}(n)$, the ACVP framework proposes a general algorithm whose mean-weight behavior is written as

$$
\mathrm{E}[\mathbf{w}(n+1)]=\mathrm{E}[\mathbf{w}(n)]+\sum_{i=1}^{3} \beta_{i}(n) \overline{\mathbf{v}}_{i}(n)
$$

where $\beta_{i}(n)$ represents the $i$ th time-varying combination coefficient [30].

The first practical outcome of the ACVP framework is the SB-ACVP algorithm, which was developed in [30] using a unipolar sigmoid function to support the adjustment of $\beta_{i}(n)$. The choice of the unipolar sigmoid function is due to its limited range (from 0 to 1 ), making it easier to control the individual contribution of each vector $\overline{\mathbf{v}}_{i}(n)$ in (17). Furthermore, the sigmoid function leads to a reduced stochastic gradient noise for values close to its range limits.

As shown in [30], the SB-ACVP exhibits low computational burden and provides higher SINR levels than the CSG-type beamforming algorithms. Table I summarizes the SB-ACVP algorithm, where $\phi\left[\alpha_{i}(n)\right]$ denotes the unipolar sigmoid function with $-\infty<\alpha_{i}(n)<\infty, \mu$ is a parameter that determines the range of $\beta_{i}(n), \mathbf{w}^{\prime}(n)$ characterizes the beamforming vector obtained before the normalization step (last step of the algorithm), and $\mathbf{I}_{K}$ represents the identity matrix with dimension $K \times K$ [30].

TABLE I

SUMMARY OF THE SB-ACVP ALGORITHM

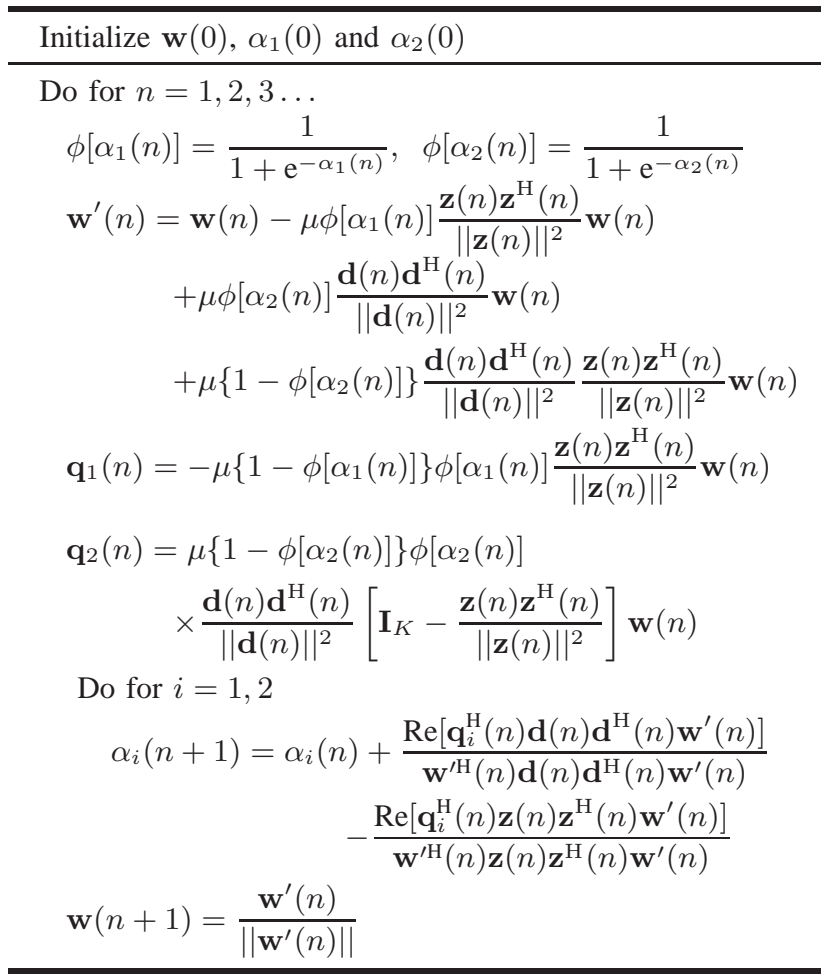




\section{Proposed Algorithm}

In order to develop the proposed beamforming algorithm, let us first introduce both unipolar and bipolar sigmoid functions for supporting the adjustment of $\beta_{i}(n)$ in (17). In contrast to the SB-ACVP [30], the proposed DS-ACVP algorithm is derived by using a bipolar sigmoid function to support the adjustment of $\beta_{1}(n)$, i.e.,

$$
\beta_{1}(n)=\mu \gamma\left[\alpha_{1}(n)\right]
$$

where $\mu$ is a parameter that specifies the range of $\beta_{1}(n)$, and $\gamma\left[\alpha_{1}(n)\right]$ denotes the bipolar sigmoid function, which is given by

$$
\gamma\left[\alpha_{1}(n)\right]=\frac{2}{1+\mathrm{e}^{-\alpha_{1}(n)}}-1
$$

with $-\infty<\alpha_{1}(n)<\infty$. One notices from (19) that $\beta_{1}(n)$ can assume both positive and negative values, which may infer that the bipolar sigmoid function is an inappropriate choice, since $\beta_{1}(n)$ is defined as a negative coefficient in both AP-CSG and AP-QCSG algorithms. However, in cases where the SOI lies in the interference subspace, negative values of $\beta_{1}(n)$ significantly reduce the projection of $\mathbf{w}(n)$ onto the SOI subspace. Thus, a positive value of $\beta_{1}(n)$ improves the SINR performance in these cases, preventing $\mathbf{w}(n)$ from being steered toward the null space of the SOI. With respect to $\beta_{2}(n)$, the strategy considered here is to use a positive range for its values, since $\overline{\mathbf{v}}_{2}(n)$ lies in the SOI subspace [30]. Then, as in the SB-ACVP (see Table I), the proposed algorithm adjusts $\beta_{2}(n)$ according to

$$
\beta_{2}(n)=\mu \phi\left[\alpha_{2}(n)\right]
$$

where

$$
\phi\left[\alpha_{2}(n)\right]=\frac{1}{1+\mathrm{e}^{-\alpha_{2}(n)}}
$$

denotes the unipolar sigmoid function. Regarding $\beta_{3}(n)$, the proposed algorithm takes into account the contribution of $\overline{\mathbf{v}}_{3}(n)$ in (17). Such a vector becomes more significant in (17) as the projection of $\mathbf{d}(n)$ onto the interference subspace increases. This characteristic of $\overline{\mathbf{v}}_{3}(n)$ is considered by the SB-ACVP algorithm to adjust $\beta_{3}(n)$ in such a way that $\mathbf{w}(n)$ is not steered toward the null space of the SOI [30]. On the other hand, the additional degree of freedom provided by the bipolar sigmoid function in (18) allows neglecting $\overline{\mathbf{v}}_{3}(n)$ in (17) without loss of algorithm performance. Thus, aiming to reduce the computational complexity, the proposed DS-ACVP algorithm is derived considering

$$
\beta_{3}(n)=0 \quad \forall n .
$$

Now, aiming to obtain the beamforming vector, we substitute (18)-(22) into (17) and eliminate the expected value from the resulting expression, leading to

$$
\begin{aligned}
\mathbf{w}(n+1)=\mathbf{w}(n)+ & \mu \gamma\left[\alpha_{1}(n)\right] \frac{\mathbf{z}(n) \mathbf{z}^{\mathrm{H}}(n)}{\|\mathbf{z}(n)\|^{2}} \mathbf{w}(n) \\
& +\mu \phi\left[\alpha_{2}(n)\right] \frac{\mathbf{d}(n) \mathbf{d}^{\mathrm{H}}(n)}{\|\mathbf{d}(n)\|^{2}} \mathbf{w}(n) .
\end{aligned}
$$

For updating $\alpha_{1}(n)$ and $\alpha_{2}(n)$ in (23), we use the steepest ascent method [33] as follows:

$$
\alpha_{i}(n+1)=\alpha_{i}(n)+\nabla f[\hat{\Gamma}(n)]
$$

with $f[\hat{\Gamma}(n)]$ denoting some arbitrary utility function that is strictly increasing with the instantaneous $\operatorname{SINR} \hat{\Gamma}(n)$. As in [30], we choose the natural logarithm function to represent such a utility function, i.e.,

$$
f[\hat{\Gamma}(n)]=\frac{1}{2} \log \left[\frac{\mathbf{w}^{\mathrm{H}}(n) \mathbf{d}(n) \mathbf{d}^{\mathrm{H}}(n) \mathbf{w}(n)}{\mathbf{w}^{\mathrm{H}}(n) \mathbf{z}(n) \mathbf{z}^{\mathrm{H}}(n) \mathbf{w}(n)}\right] .
$$

This function is strictly increasing in $\hat{\Gamma}(n)$ and allows easy computation of the gradient $\nabla f[\hat{\Gamma}(n)]$ in (24). Then, assuming that $\alpha_{i}(n)$ is updated after obtaining $\mathbf{w}(n+1),(24)$ is rewritten as

$$
\begin{array}{r}
\alpha_{i}(n+1)=\alpha_{i}(n)+\frac{\operatorname{Re}\left[\mathbf{q}_{i}^{\mathrm{H}}(n) \mathbf{d}(n) \mathbf{d}^{\mathrm{H}}(n) \mathbf{w}(n+1)\right]}{\mathbf{w}^{\mathrm{H}}(n+1) \mathbf{d}(n) \mathbf{d}^{\mathrm{H}}(n) \mathbf{w}(n+1)} \\
-\frac{\operatorname{Re}\left[\mathbf{q}_{i}^{\mathrm{H}}(n) \mathbf{z}(n) \mathbf{z}^{\mathrm{H}}(n) \mathbf{w}(n+1)\right]}{\mathbf{w}^{\mathrm{H}}(n+1) \mathbf{z}(n) \mathbf{z}^{\mathrm{H}}(n) \mathbf{w}(n+1)}
\end{array}
$$

with

$$
\mathbf{q}_{i}(n)=\frac{\partial \mathbf{w}(n+1)}{\partial \alpha_{i}(n)} .
$$

Next, substituting (23) into (27), and considering that

$$
\frac{\partial \gamma\left[\alpha_{1}(n)\right]}{\partial \alpha_{1}(n)}=\frac{1}{2}\left\{1-\gamma^{2}\left[\alpha_{1}(n)\right]\right\}
$$

and

$$
\frac{\partial \phi\left[\alpha_{2}(n)\right]}{\partial \alpha_{2}(n)}=\left\{1-\phi\left[\alpha_{2}(n)\right]\right\} \phi\left[\alpha_{2}(n)\right]
$$

$\mathbf{q}_{1}(n)$ and $\mathbf{q}_{2}(n)$ are written as follows:

$$
\mathbf{q}_{1}(n)=\mu \frac{1}{2}\left\{1-\gamma^{2}\left[\alpha_{1}(n)\right]\right\} \frac{\mathbf{z}(n) \mathbf{z}^{\mathrm{H}}(n)}{\|\mathbf{z}(n)\|^{2}} \mathbf{w}(n)
$$

and

$$
\mathbf{q}_{2}(n)=\mu \phi_{2}\left[\alpha_{2}(n)\right]\left\{1-\phi_{2}\left[\alpha_{2}(n)\right]\right\} \frac{\mathbf{d}(n) \mathbf{d}^{\mathrm{H}}(n)}{\|\mathbf{d}(n)\|^{2}} \mathbf{w}(n) .
$$

Finally, as in the SB-ACVP algorithm, the resulting $\mathbf{w}(n+1)$ is normalized aiming to avoid an unlimited growth of $\|\mathbf{w}\|$ that may arise from the vector summation present in the right-hand side of (23).

The proposed DS-ACVP algorithm is summarized in Table II. Regarding the computational complexity, Table III presents the number of real operations per iteration required by the algorithms considered in this paper. The first two terms of the Taylor series expansion are considered to obtain the computational load of the sigmoid functions given by (19) and (21). From Table III, we verify that the proposed algorithm yields a smaller complexity than both ICSG and SB-ACVP algorithms. 
TABLE II

SUMMARY OF THE DS-ACVP ALGORITHM

Initialize $\mathbf{w}(0), \alpha_{1}(0)$ and $\alpha_{2}(0)$

Do for $n=1,2,3 \ldots$

$$
\begin{aligned}
\gamma\left[\alpha_{1}(n)\right] & =\frac{2}{1+\mathrm{e}^{-\alpha_{1}(n)}}-1, \quad \phi\left[\alpha_{2}(n)\right]=\frac{1}{1+\mathrm{e}^{-\alpha_{2}(n)}} \\
\mathbf{w}^{\prime}(n)= & \mathbf{w}(n)+\mu \gamma\left[\alpha_{1}(n)\right] \frac{\mathbf{z}(n) \mathbf{z}^{\mathrm{H}}(n)}{\|\mathbf{z}(n)\|^{2}} \mathbf{w}(n) \\
& +\mu \phi\left[\alpha_{2}(n)\right] \frac{\mathbf{d}(n) \mathbf{d}^{\mathrm{H}}(n)}{\|\mathbf{d}(n)\|^{2}} \mathbf{w}(n) \\
\mathbf{q}_{1}(n)= & \frac{\mu}{2}\left\{1-\gamma^{2}\left[\alpha_{1}(n)\right]\right\} \frac{\mathbf{z}(n) \mathbf{z}^{\mathrm{H}}(n)}{\|\mathbf{z}(n)\|^{2}} \mathbf{w}(n) \\
\mathbf{q}_{2}(n)= & \mu\left\{1-\phi\left[\alpha_{2}(n)\right]\right\} \phi\left[\alpha_{2}(n)\right] \frac{\mathbf{d}(n) \mathbf{d}^{\mathrm{H}}(n)}{\|\mathbf{d}(n)\|^{2}} \mathbf{w}(n)
\end{aligned}
$$

Do for $i=1,2$

$$
\begin{array}{r}
\alpha_{i}(n+1)=\alpha_{i}(n)+\frac{\operatorname{Re}\left[\mathbf{q}_{i}^{\mathrm{H}}(n) \mathbf{d}(n) \mathbf{d}^{\mathrm{H}}(n) \mathbf{w}^{\prime}(n)\right]}{\mathbf{w}^{\prime \mathrm{H}}(n) \mathbf{d}(n) \mathbf{d}^{\mathrm{H}}(n) \mathbf{w}^{\prime}(n)} \\
-\frac{\operatorname{Re}\left[\mathbf{q}_{i}^{\mathrm{H}}(n) \mathbf{z}(n) \mathbf{z}^{\mathrm{H}}(n) \mathbf{w}^{\prime}(n)\right]}{\mathbf{w}^{\prime \mathrm{H}}(n) \mathbf{z}(n) \mathbf{z}^{\mathrm{H}}(n) \mathbf{w}^{\prime}(n)}
\end{array}
$$

$\mathbf{w}(n+1)=\frac{\mathbf{w}^{\prime}(n)}{\left\|\mathbf{w}^{\prime}(n)\right\|}$

TABLE III

COMPUTATIONAL LOAD FOR $K$ ANTENNAS

\begin{tabular}{c|c|c|c|c}
\hline \multirow{2}{*}{ Algorithm } & \multicolumn{4}{|c}{ Number of real operations per iteration } \\
\cline { 2 - 5 } & Multiplications & Additions & Divisions & $\begin{array}{c}\text { Square } \\
\text { roots }\end{array}$ \\
\hline ICSG [26] & $66 K+34$ & $52 K-8$ & 5 & 1 \\
\hline AP-CSG [28] & $28 K+6$ & $24 K-4$ & 2 & 1 \\
\hline AP-QCSG [29] & $30 K+37$ & $28 K+5$ & 3 & 2 \\
\hline SB-ACVP [30] & $46 K+77$ & $38 K+22$ & 5 & 1 \\
\hline $\begin{array}{c}\text { DS-ACVP } \\
\text { (proposed) }\end{array}$ & $40 K+57$ & $34 K+14$ & 5 & 1 \\
\hline
\end{tabular}

\section{Simulation Results}

In this section, Monte Carlo (MC) simulations (obtained from 200 independent runs) are presented to assess and compare the performance of the ICSG, AP-CSG, AP-QCSG, SBACVP, and DS-ACVP (proposed) algorithms. It is important to highlight that the algorithms considered here do not require estimating the DOA, the input autocorrelation matrix and/or the CSI.

\section{A. Simulation setup, parameters, and figures of merit}

All simulation scenarios considered here use a linear array with $K=8$ omnidirectional antennas uniformly spaced by half a wavelength. The step-size parameters are chosen aiming to provide the best performance for all algorithms, and the beamforming vectors are initialized with $\mathrm{w}=$ $\left[\begin{array}{llll}1 & 0 & \cdots & 0\end{array}\right]^{\mathrm{T}}$, which corresponds to an omnidirectional radiation pattern over the azimuth plane. In all simulations, the normalized power (relative to the noise power) of the signals arriving at the $\mathrm{BS}$ is fixed in $30 \mathrm{~dB}$ for the $\mathrm{SOI}$ and $20 \mathrm{~dB}$ for the interfering signals (coming from co-channel cells). Moreover, a Rayleigh channel with $L=12$ independent fading paths is considered, giving rise to an angle spread of $5^{\circ}$ around the angle of arrival of each user.

With respect to the figures of merit considered in the simulations, we evaluate the SINR [given by (7)] during the iterative process and the radiation pattern in the last iteration of the corresponding algorithm. In addition, the sigmoid function curves related to the DS-ACVP algorithm are presented aiming to verify the effectiveness of the proposed approach for adjusting the combination coefficients. All curves are obtained by averaging the Monte Carlo runs.

\section{B. Experimental results}

Example 1: In this example, we assess the performance of the beamforming algorithms in a scenario with low interference levels, in which the DOA of the SOI is quite different from the DOAs of the interfering signals. The scenario consists of a SOI located at $0^{\circ}$ and two interfering signals located at $30^{\circ}$ and $-30^{\circ}$. The results of this example are shown in Fig. 1. From the SINR results presented in Fig. 1(a), one can notice that the proposed DS-ACVP algorithm outperforms the ICSG, AP-CSG, and AP-QCSG algorithms. Moreover, the DS-ACVP provides faster convergence rate and similar steady-state SINR as compared with the SB-ACVP algorithm. The radiation patterns illustrated in Fig. 1(b) show that both SB-ACVP and DS-ACVP algorithms exhibit the best tradeoff between the gain in the SOI direction (indicated by 0 ) and the attenuation in the direction of the interferers (indicated by $x)$. In addition, the curves corresponding to the evolution of $\gamma\left[\alpha_{1}(n)\right]$ and $\phi\left[\alpha_{2}(n)\right]$ are shown in Fig. 1(c). One observes from these curves that the unipolar sigmoid function $\phi\left[\alpha_{2}(n)\right]$ grows at the initial transient phase aiming to increase the projection of $\mathbf{w}(n)$ onto the SOI subspace. Hereafter, $\phi\left[\alpha_{2}(n)\right]$ and $\gamma\left[\alpha_{1}(n)\right]$ are decreased down to their lower bounds, thus steering $\mathbf{w}(n)$ towards the null space of the interfering signals. Such a behavior ratifies the effectiveness of the proposed strategy for obtaining coefficients $\beta_{1}(n)$ and $\beta_{2}(n)$.

Example 2: In this second example, the SOI and one interferer arrive at the BS with nearby angles-of-arrival, thus allowing to assess the spatial filtering capability of the algorithms. In such a scenario, the SOI is located at $45^{\circ}$ and the two interfering signals are located at $40^{\circ}$ and $50^{\circ}$. The curves obtained in this example are depicted in Fig. 2. From the SINR results shown in Fig. 2(a), one notices that the steady-state SINR levels achieved by all algorithms are lower than those of the previous example, which is due to the proximity of the involved signals. Nevertheless, the proposed DS-ACVP leads to higher SINR levels as compared with the other algorithms considered here. The radiation patterns presented in Fig. 2(b) also confirm a better performance of the proposed algorithm. In addition, as compared with the results obtained in Example 1, one observes from Fig. 2(c) that $\gamma\left[\alpha_{1}(n)\right]$ takes longer to achieve its lower bound and $\phi\left[\alpha_{2}(n)\right]$ does not reach zero. This behavior confirms the proper operation of the proposed algorithm, since $\overline{\mathbf{v}}_{1}(n)$ contains vector components that lie in 
the SOI subspace (due to the SOI-interferer proximity) and a higher contribution of $\overline{\mathbf{v}}_{2}(n)$ has been taking into account for preventing SOI suppression.

Example 3: In this example, we consider a scenario with high interference levels in which the SOI and one interferer arrive at the BS with the same DOA. Specifically, the SOI and the first interfering signal are located at $-30^{\circ}$, whereas the second interferer is located at $30^{\circ}$. This is a challenging scenario due to the intersection between the subspaces spanned by the involved signals. Therefore, any unbalanced behavior of the beamforming algorithm [26] results in the suppression of the SOI. The results of this example are illustrated in Fig. 3. As shown in Figs. 3(a) and (b), all algorithms lead to the same steady-state SINR levels and present very similar radiation patterns. Such a result indicates that the proposed algorithm in severe conditions prevents SOI suppression as is done by SB-ACVP and CSG-type algorithms. The sigmoid function curves shown in Fig. 3(c) confirm that the combination coefficients are adjusted aiming to attenuate the interferers without reducing the gain in the SOI direction.

Example 4: The aim of this example is to verify the tracking capability of the algorithms. The scenario is composed of an SOI located at $60^{\circ}$, and two interferers arriving with DOAs of $60^{\circ}$ and $-60^{\circ}$. Then, after 400 iterations, the DOA of the SOI is changed to $30^{\circ}$. After this change, the increase of the difference between the DOAs allows enhancing the SINR at the array output. This behavior is noticed in Fig. 4, in which the curves of SINR presented in Fig. 4(a) confirm that both SB-ACVP and DS-ACVP outperform the CSG-type algorithms after changing the SOI direction. Figs. 4(b) and (c) show the radiation patterns at iteration $n=400$ (immediately before changing the DOA) and iteration $n=800$, illustrating the tracking capability of the algorithms considered here. In addition, one notices from Fig. 4(d) that the values of $\gamma\left[\alpha_{1}(n)\right]$ and $\phi\left[\alpha_{2}(n)\right]$ are properly adjusted before and after changing the SOI direction.

Example 5: In this example, we consider a scenario in which an additional interferer is added after the convergence of the algorithms. Specifically, the scenario is initialized with a SOI located at $45^{\circ}$ and three interferers located at $-30^{\circ}, 0^{\circ}$, and $80^{\circ}$. Then, after 750 iterations, another interfering signal is added at $60^{\circ}$. Such an interferer acts as a disturbance in the convergence process, allowing to test both stability and adaptability of the algorithms. The ICSG algorithm is not included in this example, since it has been originally formulated for scenarios with two interferers. The curves obtained in this example are depicted in Fig. 5. One observes from Fig. 5(a) that the algorithms based on the ACVP framework outperform the CSG-type algorithms considered in this example. As compared with the SB-ACVP, the proposed DS-ACVP achieves a slightly larger SINR before the iteration $n=750$ and provides a faster convergence rate after adding the fourth interferer. The radiation patterns shown in Figs. 5(b) and (c) confirm a better performance of the proposed algorithm. Moreover, the curves of $\gamma\left[\alpha_{1}(n)\right]$ and $\phi\left[\alpha_{2}(n)\right]$ presented in Fig. 5(d) point out that the interferer added at $n=750$ has no influence on the adjustment of the combination coefficients. Such a behavior is expected due to the large difference between the DOA of the
SOI and of the new interfering signal.

Example 6: In this last example, we consider a scenario with high interference levels where the number of interferers is larger than the number of antennas. This is a challenging scenario, since the number of antennas is insufficient to place nulls in the direction of the interfering signals, i.e. $K<M-1$ [1]. We assume here a SOI located at $45^{\circ}$ and nine interferers located at $-90^{\circ},-70^{\circ},-50^{\circ},-30^{\circ}, 0^{\circ}, 30^{\circ}, 60^{\circ}, 80^{\circ}$, and $90^{\circ}$. The results of this example are illustrated in Fig. 6. As shown in Fig. 6(a), the proposed DS-ACVP algorithm provides the best SINR performance among the algorithms assessed in this example. The resulting radiation patterns, presented in Fig. 6(b), corroborate the steady-state SINR achieved by the beamforming algorithms. In addition, the evolution of the sigmoid functions shown in Fig. 6(c) illustrates the dynamic adjustment of $\beta_{1}(n)$ and $\beta_{2}(n)$ provided by the proposed DSACVP algorithm.

\section{Discussion}

The examples presented in this section show that the proposed DS-ACVP outperforms the CSG-type algorithms and can provide equal or higher SINR than the SB-ACVP algorithm. In particular, Example 1 shows that both SB-ACVP and DS-ACVP achieve considerably higher SINR levels as compared with the CSG-type algorithms. Example 2 verifies the ability of the proposed DS-ACVP to suppress interference in scenarios where the SOI is close to an interfering signal, thus outperforming all considered algorithms. Example 3 confirms that the strategy used to adjust the combination coefficients does not result in an unbalanced behavior of the proposed DS-ACVP algorithm, which maintains a sufficient gain toward the SOI even when there is an interference located at this same direction. Example 4 shows that the proposed DS-ACVP performs well in nonstationary scenarios and is capable of tracking variations in the DOA of the SOI. Example 5 corroborates the effectiveness of the proposed approach to readjust the beamforming vector, aiming to suppress the third interferer added after the convergence. Finally, we verify in Example 6 that the proposed algorithm is effective to improve the SINR in scenarios with high interference levels, outperforming the competing algorithms considered here.

\section{CONCLUding REMARKS}

In this paper a new beamforming algorithm was introduced based on the ACVP framework discussed in [30]. Such an algorithm, called DS-ACVP, considers both unipolar and bipolar sigmoid functions for controlling the excursion of the combination coefficients involved in the ACVP framework. This strategy allows reducing the number of vector projections required in the beamforming update. As a result, the proposed DS-ACVP algorithm presents a lower computational complexity than the SB-ACVP algorithm, while maintaining a competing SINR for performance. Computer results confirmed the effectiveness of the proposed algorithm. 


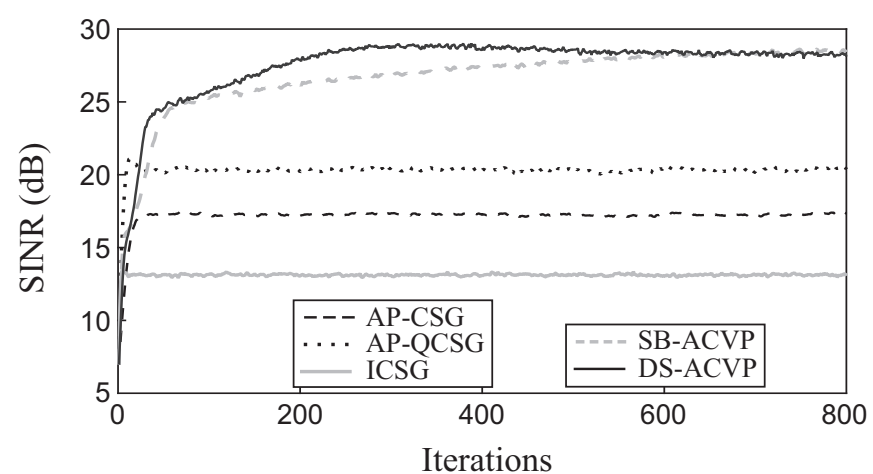

(a)

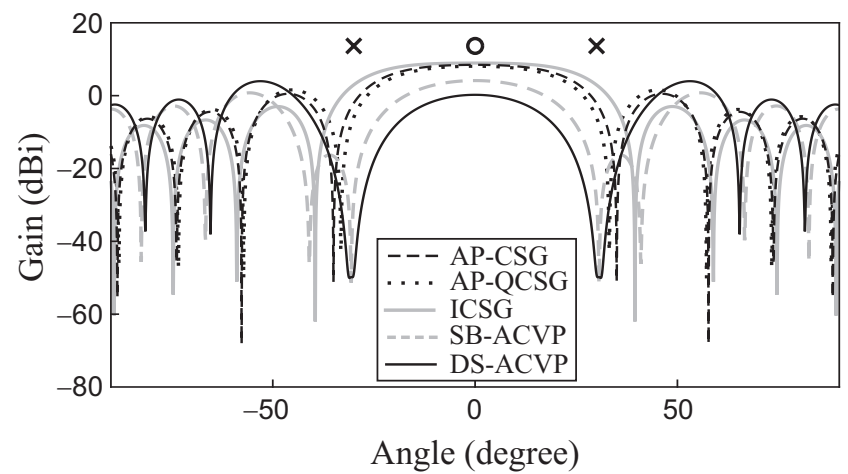

(b)

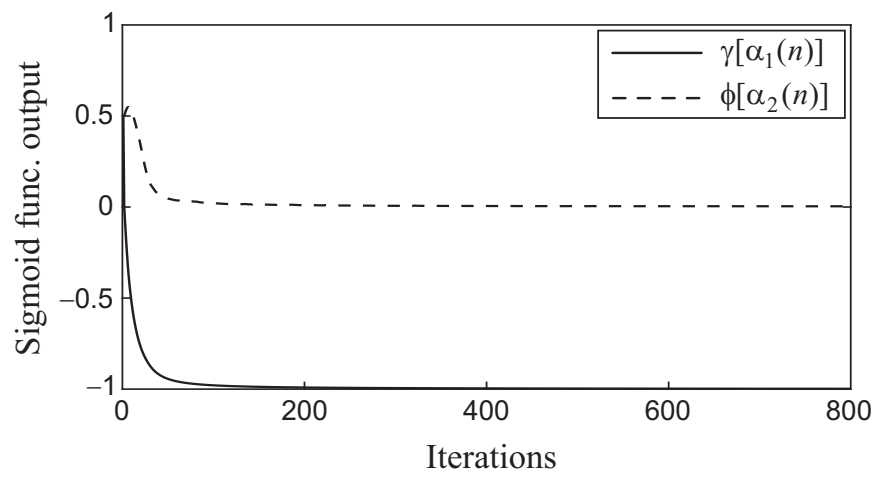

(c)

Fig. 1. Example 1. (a) SINR comparison. (b) Radiation pattern comparison. (c) Mean behavior of the sigmoid functions $\gamma\left[\alpha_{1}(n)\right]$ and $\phi\left[\alpha_{2}(n)\right]$ used by the proposed DS-ACVP algorithm.

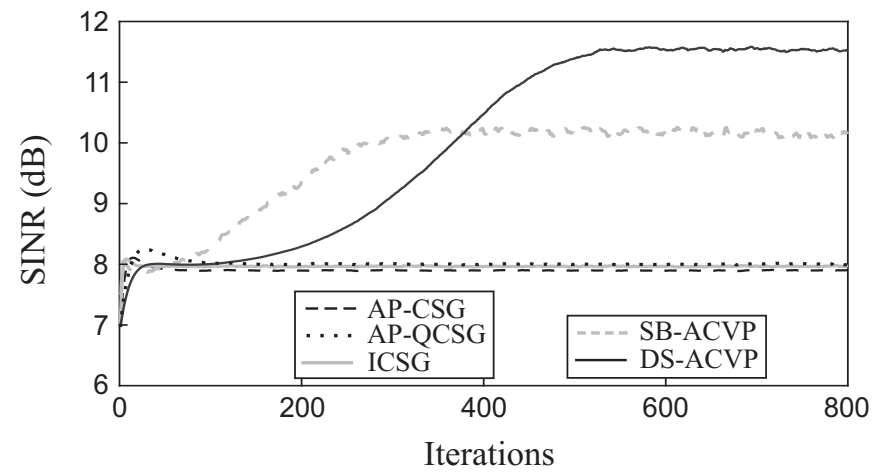

(a)

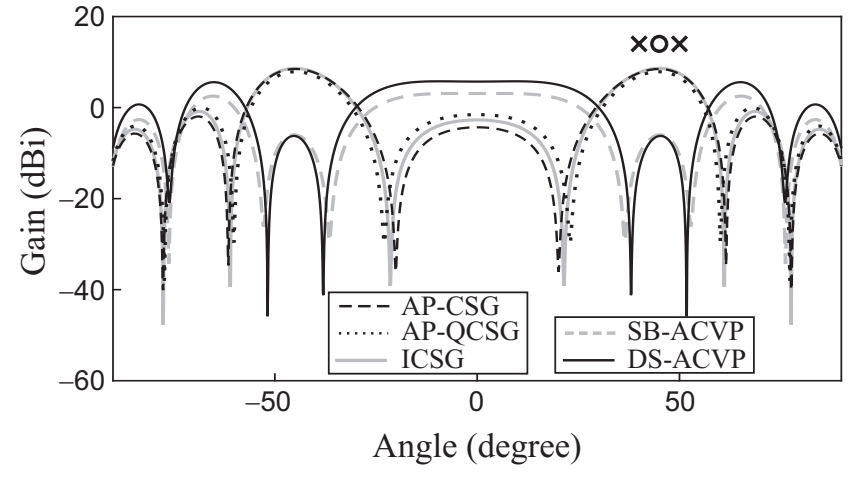

(b)

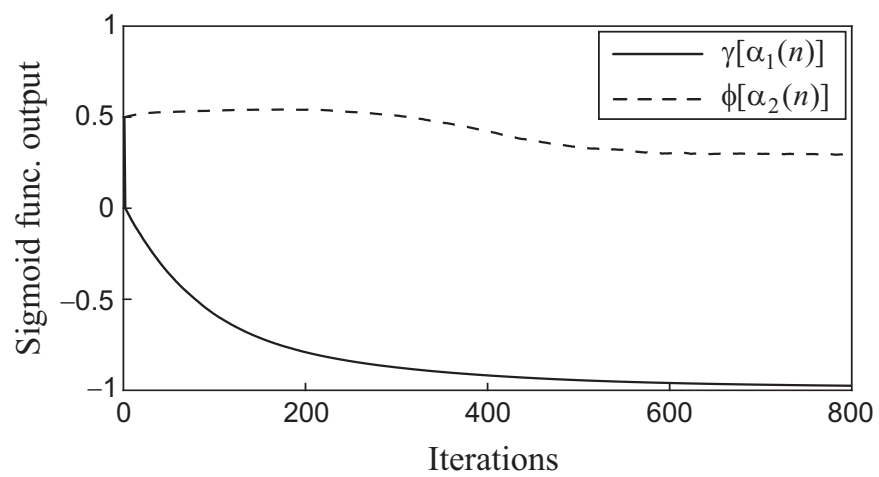

(c)

Fig. 2. Example 2. (a) SINR comparison. (b) Radiation pattern comparison. (c) Mean behavior of the sigmoid functions $\gamma\left[\alpha_{1}(n)\right]$ and $\phi\left[\alpha_{2}(n)\right]$ used by the proposed DS-ACVP algorithm. 


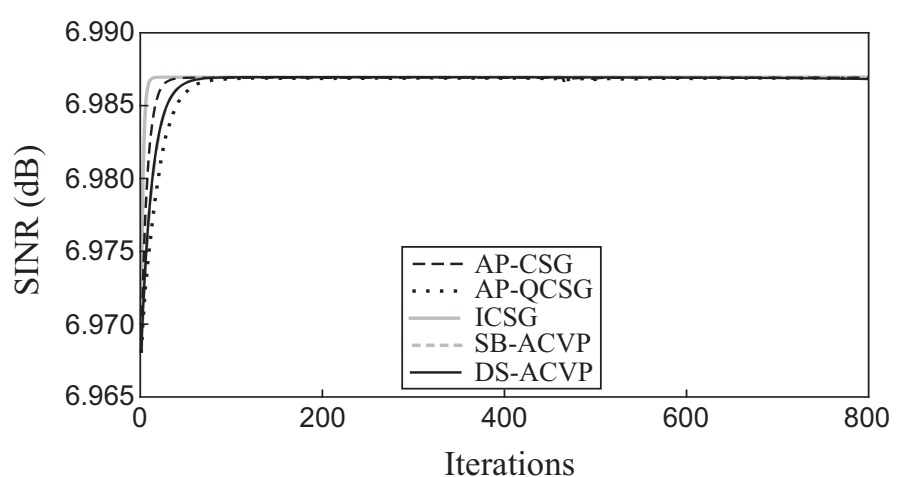

(a)

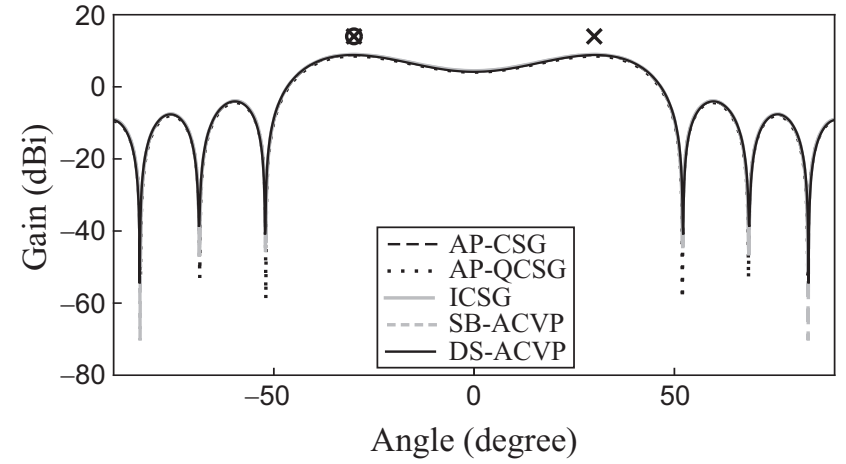

(b)

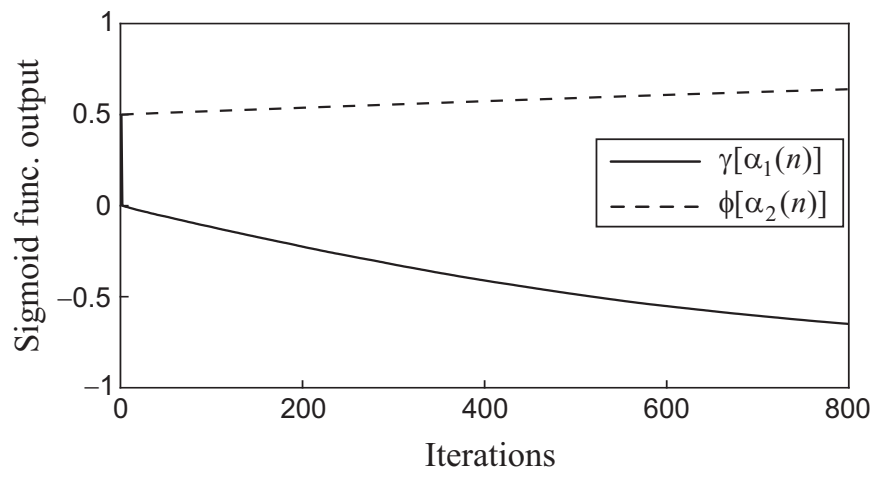

(c)

Fig. 3. Example 3. (a) SINR comparison. (b) Radiation pattern comparison. (c) Mean behavior of the sigmoid functions $\gamma\left[\alpha_{1}(n)\right]$ and $\phi\left[\alpha_{2}(n)\right]$ used by the proposed DS-ACVP algorithm.
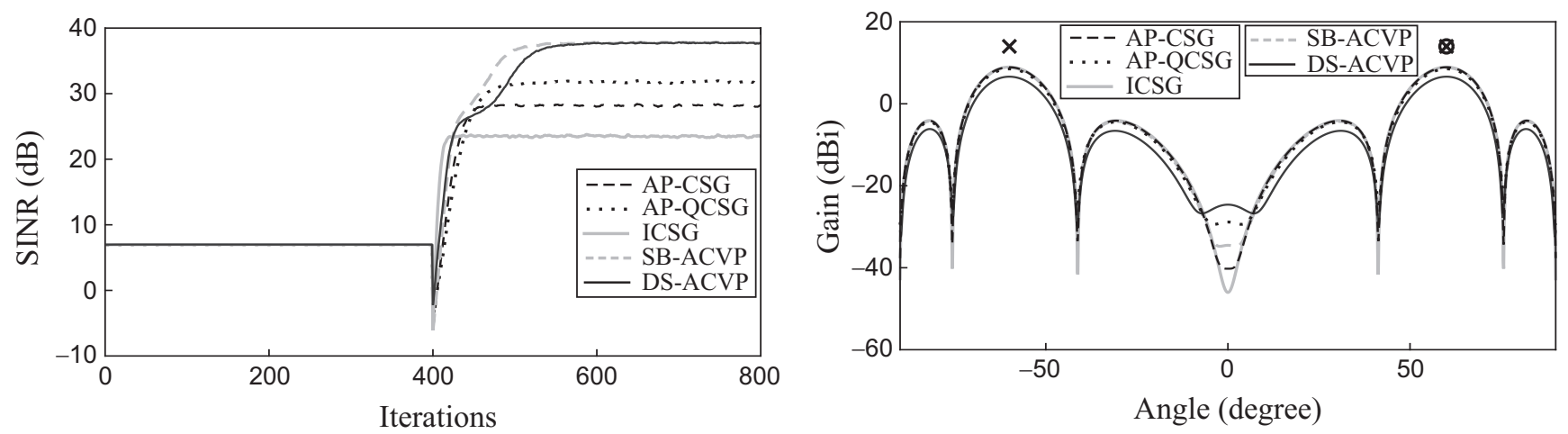

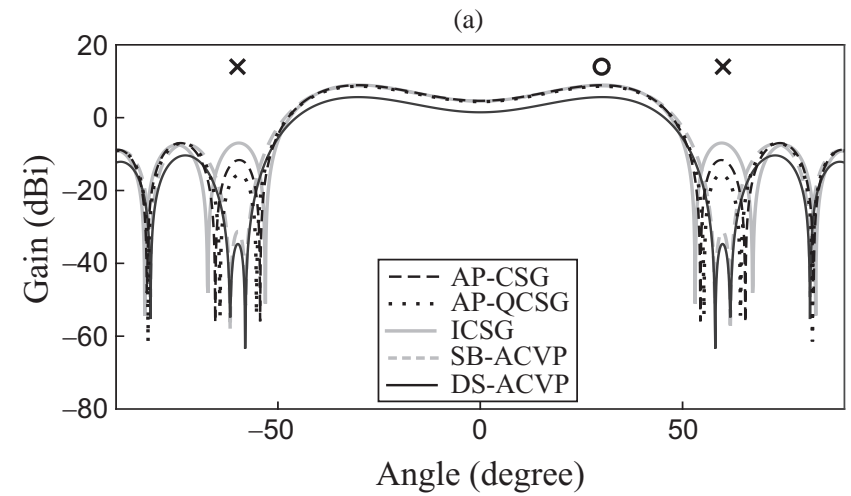

(c)

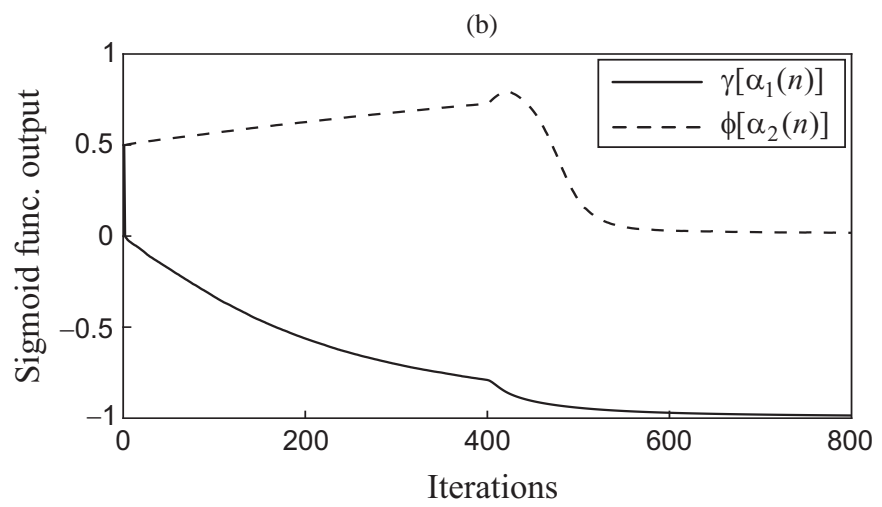

(d)

Fig. 4. Example 4. (a) SINR comparison. (b) Radiation pattern comparison at the iteration $n=400$. (c) Radiation pattern comparison at $n=800$. (d) Mean behavior of the sigmoid functions $\gamma\left[\alpha_{1}(n)\right]$ and $\phi\left[\alpha_{2}(n)\right]$ used by the proposed DS-ACVP algorithm. 

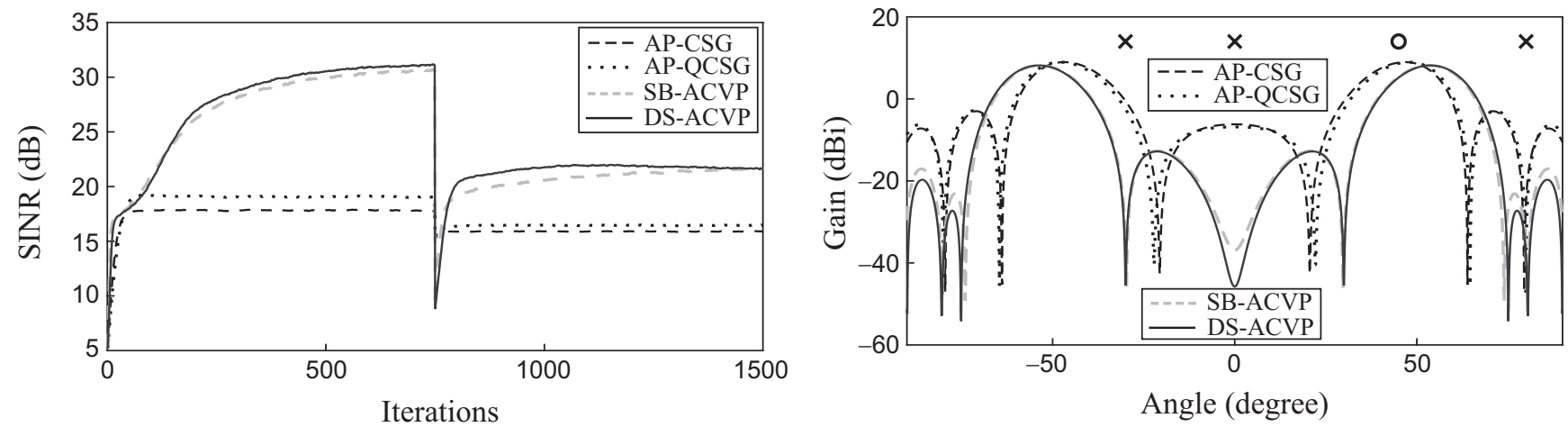

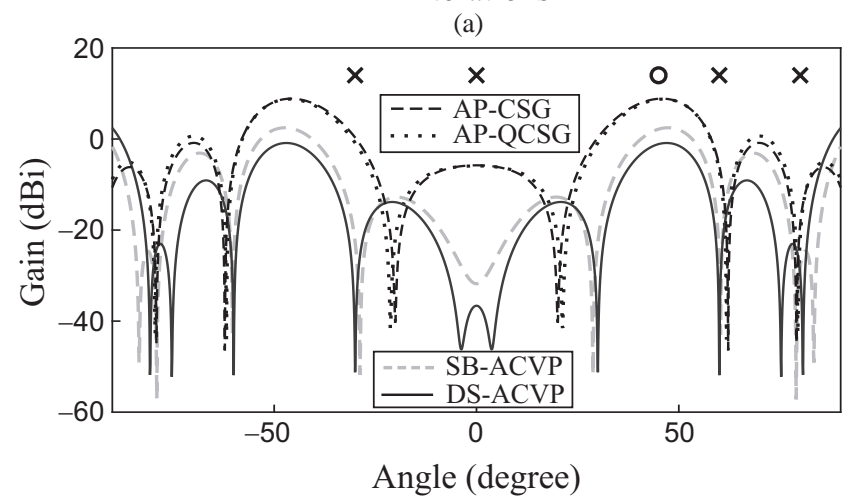

(c)

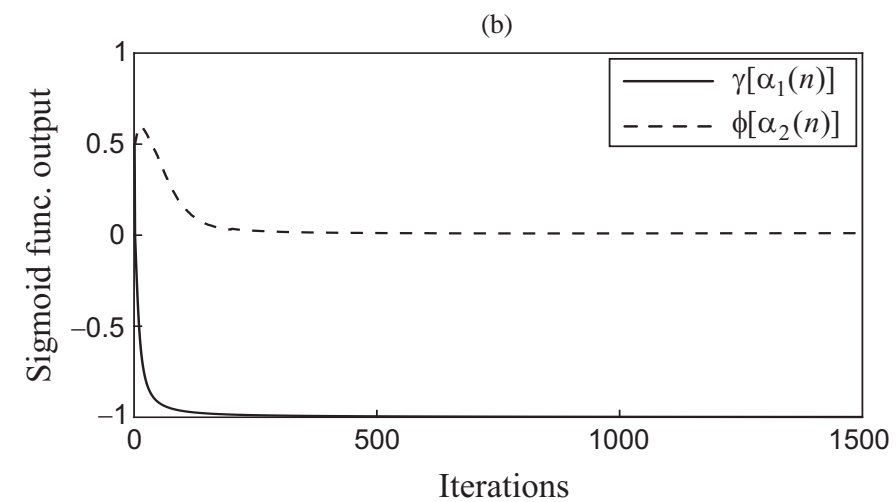

(d)

Fig. 5. Example 5. (a) SINR comparison. (b) Radiation pattern comparison at the iteration $n=750$. (c) Radiation pattern comparison at $n=1500$. (d) Mean behavior of the sigmoid functions $\gamma\left[\alpha_{1}(n)\right]$ and $\phi\left[\alpha_{2}(n)\right]$ used by the proposed DS-ACVP algorithm.

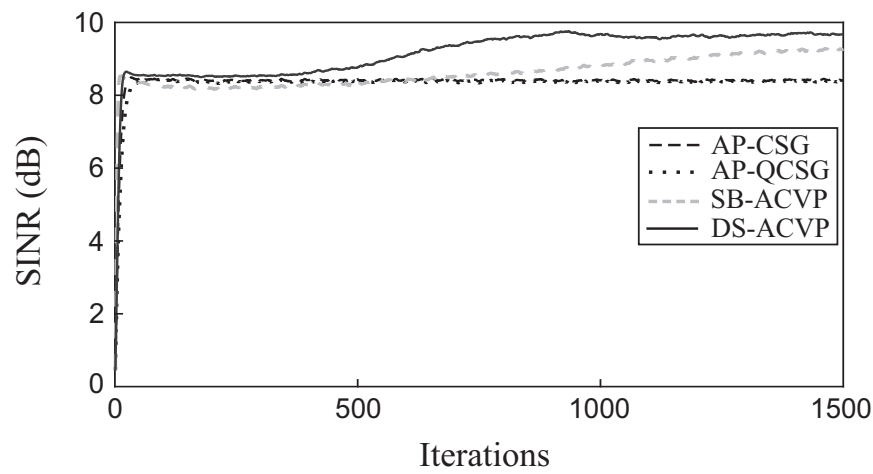

(a)

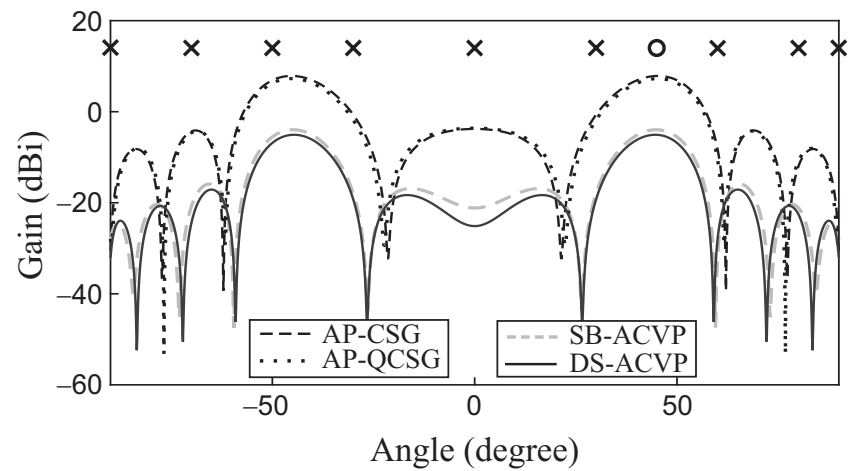

(b)

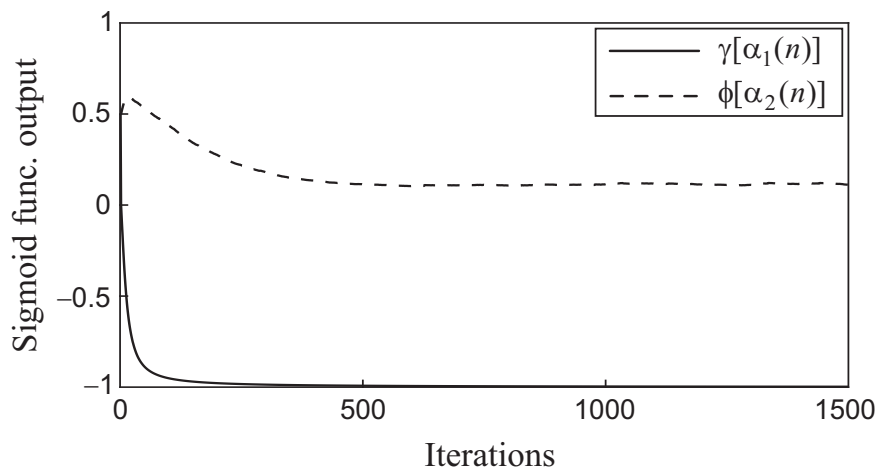

(c)

Fig. 6. Example 6. (a) SINR comparison. (b) Radiation pattern comparison. (c) Mean behavior of the sigmoid functions $\gamma\left[\alpha_{1}(n)\right]$ and $\phi\left[\alpha_{2}(n)\right]$ used by the proposed DS-ACVP algorithm. 


\section{REFERENCES}

[1] C. A. Balanis and P. I. Ioannides, Introduction to Smart Antennas, San Rafael, CA, USA: Morgan \& Claypool Publishers, 2007, doi: 10.2200/S00079ED1V01Y200612ANT005.

[2] L. C. Godara, Smart Antennas. Boca Raton, FL: CRC Press, 2004.

[3] M. K. Karakayali, G. J. Foschini, and R. A. Valenzuela, "Network coordination for spectrally efficient communications in cellular systems," IEEE Wireless Commun., vol. 13, no. 4, pp. 56-61, Aug. 2006, doi: 10.1109/MWC.2006.1678166.

[4] L. C. Godara, Handbook of Antennas in Wireless Communications. Boca Raton, FL: CRC Press, 2002.

[5] B. Widrow, P. E. Mantey, L. J. Griffiths, and B. B. Goode, "Adaptive antenna systems," Proc. IEEE, vol. 55, no. 12, pp. 2143-2159, Dec. 1967, doi: 10.1109/PROC.1967.6092.

[6] F. Molisch, Wireless Communications. Chichester: John Wiley \& Sons, 2010.

[7] J. A. Zhang, T. T. Yang, and Z. Chen, "Under-determined training and estimation for distributed transmit beamforming systems," IEEE Trans. Wireless Commun., vol. 12, no. 4, pp. 1936-1946, Apr. 2013, doi: 10.1109/TWC.2013.022013.121258.

[8] S. Noh, M. D. Zoltowski, and D. J. Love, "Training sequence design for feedback assisted hybrid beamforming in massive MIMO systems," IEEE Trans. Commun., vol. 64, no. 1, pp. 187-200, Jan. 2016, doi: 10.1109/TCOMM.2015.2498184.

[9] J. Chen, H. Yin, L. Cottatellucci, and D. Gesbert, "Feedback mechanisms for FDD massive MIMO with D2D-based limited CSI sharing," IEEE Trans. Wireless Commun., vol. 16, no. 8, pp. 5162-5175, Aug. 2017, doi: 10.1109/TWC.2017.2706279

[10] P. N. Alevizos, X. Fu, N. D. Sidiropoulos, Y. Yang, and A. Bletsas, "Limited feedback channel estimation in massive MIMO with nonuniform directional dictionaries," IEEE Trans. Signal Process., vol. 66, no. 19, pp. 5127-5141, Oct. 2018, doi: 10.1109/TSP.2018.2865412.

[11] O. L. Frost, "An algorithm for linearly constrained adaptive array processing," Proc. IEEE, vol. 60, no. 8, pp. 926-935, Aug. 1972, doi: 10.1109/PROC.1972.8817

[12] S. A. Vorobyov, H. Chen, and A. B. Gershman, "On the relationship between robust minimum variance beamformers with probabilistic and worst-case distortionless response constraints," IEEE Trans. Signal Process., vol. 56, no. 11, pp. 5719-5724, Nov. 2008, doi: 10.1109/TSP.2008.929866.

[13] A. B. Gershman, N. D. Sidiropoulos, and S. Shahbazpanahi, "Convex optimization-based beamforming: from receive to transmit and network designs," IEEE Signal Process. Mag., vol. 27, no. 3, pp. 62-75, May 2010, doi: 10.1109/MSP.2010.936015.

[14] L. Zhang, W. Liu, and L. Yu, "Performance analysis for finite sample MVDR beamformer with forward backward processing," IEEE Trans. Signal Process., vol. 59, no. 5, pp. 2427-2431, May 2011, doi: 10.1109/TSP.2011.2109957.

[15] S. A. Vorobyov, "Principles of minimum variance robust adaptive beamforming design," Signal Process., vol. 93, no. 12, pp. 3264-3277, Dec. 2013, doi: 10.1016/j.sigpro.2012.10.021.

[16] S. A. Vorobyov, A. B. Gershman, Z.-Q. Luo, and N. Ma, "Adaptive beamforming with joint robustness against mismatched signal steering vector and interference nonstationarity," IEEE Signal Process. Lett., vol. 11, no. 2, pp. 108-111, Feb. 2004, doi: 10.1109/LSP.2003.819857.

[17] R. G. Lorenz and S. P. Boyd, "Robust minimum variance beamforming," IEEE Trans. Signal Process., vol. 53, no. 5, pp. 1684-1696, May 2005, doi: 10.1109/TSP.2005.845436.

[18] S. J. Kim, A. Magnani, A. Mutapcic, S. P. Boyd, and Z. Q. Luo, "Robust beamforming via worst-case SINR maximization," IEEE Trans. Signal Process., vol. 56, no. 4, pp. 1539-1547, Apr. 2008, doi: 10.1109/TSP.2007.911498.

[19] S. Yi, Y. Wu, and Y. Wang, "Projection-based robust adaptive beamforming with quadratic constraint," Signal Process., vol. 122, pp. 65-74, May 2016, doi: 10.1016/j.sigpro.2015.11.016.

[20] L. Zhang, B. Li, L. Huang, T. Kirubarajan, and H. C. So, "Robust minimum dispersion distortionless response beamforming against fast-moving interferences," Signal Process., vol. 140, pp. 190-197, Nov. 2017, doi: 10.1016/j.sigpro.2017.04.019.

[21] X. Wang, M. Amin, and X. Wang, "Robust sparse array design for adaptive beamforming against DOA mismatch," Signal Process., vol. 146, pp. 41-49, May 2018, doi: 10.1016/j.sigpro.2017.12.018.

[22] S. Choi and D. Shim, "A novel adaptive beamforming algorithm for a smart antenna system in a CDMA mobile communication environment," IEEE Trans. Veh. Technol., vol. 49, no. 5, pp. 1793-1806, Sep. 2000, doi: $10.1109 / 25.892584$.
[23] S. Choi, J. Choi, Heung-Jae Im, and B. Choi, "A novel adaptive beamforming algorithm for antenna array CDMA systems with strong interferers," IEEE Trans. Veh. Technol., vol. 51, no. 5, pp. 808-816, Sep. 2002, doi: 10.1109/TVT.2002.801546.

[24] J. Yang, H. Xi, F. Yang, and Y. Zhao, "Fast adaptive blind beamforming algorithm for antenna array in CDMA systems," IEEE Trans. Veh. Technol., vol. 55, no. 2, pp. 549-558, Mar. 2006, doi: 10.1109/TVT.2005.863419.

[25] D. R. Morgan, "Downlink adaptive array algorithms for cellular mobile communications," IEEE Trans. Commun., vol. 51, no. 3, pp. 476-488, Mar. 2003, doi: 10.1109/TCOMM.2003.809783.

[26] J. Kolodziej, O. J. Tobias, R. Seara, and D. R. Morgan, "On the constrained stochastic gradient algorithm: model, performance, and improved version," IEEE Trans. Signal Process., vol. 57, no. 4, pp. 1304-1315, Apr. 2009, doi: 10.1109/TSP.2008.2010375.

[27] G. G. Raleigh and V. K. Jones, "Adaptive antenna transmission for frequency duplex digital wireless communication," in Proc. IEEE Int. Conf. Communications, Montreal, Canada, Jun. 1997, vol. 2, pp. 641-646, doi: 10.1109/ICC.1997.609909.

[28] C. A. Pitz, E. L. O. Batista, and R. Seara, "On the joint beamforming and power control in cellular systems: algorithm and stochastic model," IEEE Trans. Wireless Commun., vol. 13, no. 12, pp. 6943-6954, Dec. 2014, doi: 10.1109/TWC.2014.2359021.

[29] C. A. Pitz, E. Batista, and R. Seara, "A quadratically-constrained stochastic gradient algorithm for beamforming in mobile communications," IEEE Trans. Circuits Syst. II, Exp. Briefs, vol. 65, no. 1, pp. 125-129, Jan. 2018, doi: 10.1109/TCSII.2017.2694338.

[30] C. A. Pitz, "Adaptive algorithms for beamforming and power control in mobile communication systems," (in Portuguese). Ph.D. dissertation, Dep. Electr. Electron. Eng., Federal University of Santa Catarina, Florianópolis, SC, Brazil, 2015.

[31] S.-K. Ahn and K. Yang, "Adaptive modulation and coding schemes based on LDPC codes with irregular modulation," IEEE Trans. Commun., vol. 58, no. 9, pp. 2465-2470, Sep. 2010, doi: 10.1109/TCOMM.2010.080310.090176.

[32] W. Jiao, H. Ding, H. Wu, and G. Yu, "Spectrum efficiency of jointing adaptive modulation coding and truncated ARQ with QOS constraints," IEEE Access, vol. 6, pp. 46915-46925, Aug. 2018, doi: 10.1109/ACCESS.2018.2867109.

[33] S. Haykin, Adaptive Filter Theory. 5th ed., Pearson, Upper Saddle River, NJ, 2014.

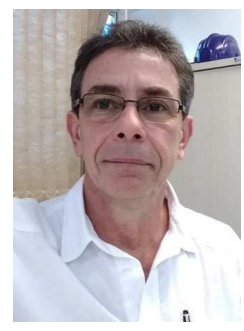

Raimundo N. G. Robert was born in Belém, PA, Brazil. He received the BS degree in electrical engineering from Federal University of Pará in 1986 and the M.Sc. degree from the Federal University of Santa Catarina in 1989. He is currently working toward the Ph.D. degree at the LINSE-Signal Processing and Circuits Laboratory, Federal University of Santa Catarina, Brazil. He joined the Electrical Engineering Department at the State University of Santa Catarina, Brazil, in 1990, where he is currently a Professor. His research interests include signal processing, applications of adaptive filtering in communication systems, and technology in education.

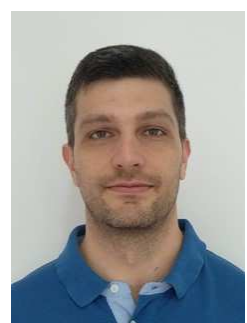

Ciro André Pitz was born in Gaspar, SC, Brazil. $\mathrm{He}$ received the B.S. degree in telecommunication engineering and the M.Sc. degree in electrical engineering from the Regional University of Blumenau, Brazil, in 2008 and 2010, respectively. In 2015, he received the $\mathrm{Ph} . \mathrm{D}$. degree in electrical engineering from Federal University of Santa Catarina, Brazil. From 2015 to 2017, he was a postdoctoral researcher at the LINSE-Circuits and Signal Processing Laboratory, Federal University of Santa Catarina, Brazil. In 2018, he joined the Department of Control, Automation and Computational Engineering at the Federal University of Santa Catarina, Brazil, where he is currently a Professor. His present research interests include adaptive signal processing theory and its application in communication systems. 


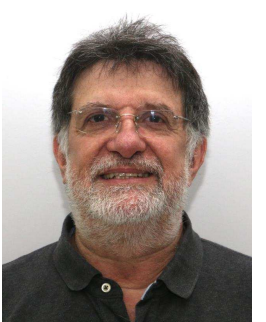

Rui Seara was born in Florianópolis, SC, Brazil. He received the B.S. and M.Sc. degrees in electrical engineering from Federal University of Santa Catarina, Brazil, in 1975 and 1980, respectively. In 1984, he received the Doctoral degree in Electrical Engineering from the Paris-Sud University, Paris, France. He joined the Electrical Engineering Department at the Federal University of Santa Catarina, Brazil, in 1976, where he is currently a Professor of Electrical Engineering, and Director of LINSE-Circuits and Signal Processing Laboratory. His research interests include digital and analog filtering, adaptive signal processing algorithms, image and speech processing, and digital communications. 